\title{
Modeling principal stress rotation for anisotropic sand
}

\author{
Yu-Xia Kong ${ }^{\text {i) }}$, Yang-Ping Yao ${ }^{\text {ii) }}$ and Ji-Dong Zhao ${ }^{\text {iii) }}$
}

\begin{abstract}
i) Lecture, Institute of Geotechnical Engineering, Nanjing Tech University, 200 North Zhongshan Road, Nanjing 210009, China. ii) Professor, Department of Civil Engineering, Beihang University, 37 Xueyuan Road, Haidian District, Beijing 100191, China. iii) Associate Professor, Department of Civil and Environmental Engineering, The Hong Kong University of Science and Technology, Hong Kong, China.
\end{abstract}

\begin{abstract}
This paper extends a constitutive model previously developed by the authors to model the anisotropic sand behavior during cyclic rotation of principal stress axes. A new anisotropic stress tensor is defined by transforming the principal stresses during the rotation, and is then incorporated to formulate the incremental form of the constitutive relation for anisotropic soil subjected to cyclic load. All the model parameters can be conveniently calibrated by triaxial compression/extension tests. The new model is validated by simulating the behavior of Toyoura sand subjected to rotational shearing and good comparison is observed with the experimental data.
\end{abstract}

Keywords: anisotropy, principal stress rotation, constitutive model, sand

\section{INTRODUCTION}

The rotation of principal stress direction in sand is a commonly encountered loading condition associated with important practical geotechnical applications. Typical examples include the soil at the foundation of offshore structures such as oil rigs and wind turbines subjected to cyclic wave load, and the rail track foundation subjected to repeated wheel load. The impact of earthquake load may well exemplify the rotation of principal stress direction within a relatively short duration. It has been recognized that the behaviour of sand depends on the principal stress direction, due primarily to inherent anisotropy. The apparent influence of anisotropy on the sand response during the rotation of principal stress direction has been observed in many studies (Symes et al. 1984; Miura et al. 1986; Sivathayalan and Vaid 2002). Principal stress rotation may result in considerable accumulation of plastic strains in sand, and generate excess pore water pressure under undrained condition (Arthur et al. 1979; Hicher and Lade 1987; Nakata et al. 1998).

In the case of stress rotation, the liquefaction resistance of sand can be reduced as much as 10-30\% as compared to the case without rotation (Ishihara and Towhata 1983; Ishihara and Yamazaki 1984). Meanwhile, experimental data (Miura et al. 1986; Symes et al. 1988) show that the behaviour of both the shear and volumetric deformation of sand can be changed by the rotation of principal stress axes. This has indeed been confirmed by some recent hollow cylinder torsional shear tests on anisotropic sand (Yang et al.
2007; Tong et al. 2010). Significant permanent strain has also been observed in the tests on principal stress rotations relevant to track foundation by Grabe and Clayton (2009).

The current paper aims to develop a constitutive model to account for the effect of principal stress rotation on the behaviour of anisotropic sand. This study builds on a previous work by the authors (Yao and Kong 2012). An extended UH (Unified Hardening) model has been developed in Yao and Kong (2012) to consider the influence of inherent anisotropy, Based on the concept of spatially mobilized planes originally proposed by Matsuoka (1974) and Matsuoka (1976). However, the extended UH model has difficulty to predict the plastic deformation correctly for the case of principal stress rotation, e.g., the model will predict no plastic strain in this case at all. To address this issue, a stress transformation method will be proposed in this paper to extend the UH model to account for the effect of stress rotation. The incremental relation is then formulated directly for the strain and the transformed anisotropic stress. The new model is further validated by comparison with experimental data.

\section{STRESS TRANSFORMATION}

\subsection{Test of Principal Stress Axes Rotation}

Hollow cylinder rotational shear apparatus (Fig. 1) has been commonly used to investigate the effects of principal stress rotation during shear of sand (Hight et al. 1983; Miura et al. 1986; Symes et al. 1984; Symes et al. 1988; Vaid et al. 1990; Tong et al. 2010). The four 
stress components, i.e. the radial stress $\sigma_{r}$, the circumferential stress $\sigma_{\theta}$ and the torsional shear stress $\sigma_{z \theta}$, can be independently applied on a typical soil specimen in such tests. In Fig. $1, \beta$ denotes the rotation angle of the principal stress axes. Figure 2 depicts the stress path in the deviatoric stress plane for a pure principal stress axes rotation test (only rotation angle changes). For specimens tested under pure principal stress rotation, the four stress components can be expressed by the principal stresses $\left(\sigma_{1}, \sigma_{2}, \sigma_{3}\right)$ and the rotation angle $\beta$ as follows:

$$
\begin{gathered}
\sigma_{z}=\frac{\sigma_{1}+\sigma_{3}}{2}+\frac{\sigma_{1}-\sigma_{3}}{2} \cos 2 \beta \\
\sigma_{\theta}=\frac{\sigma_{1}+\sigma_{3}}{2}-\frac{\sigma_{1}-\sigma_{3}}{2} \cos 2 \beta \\
\sigma_{z \theta}=\frac{\sigma_{1}-\sigma_{3}}{2} \sin 2 \beta
\end{gathered}
$$

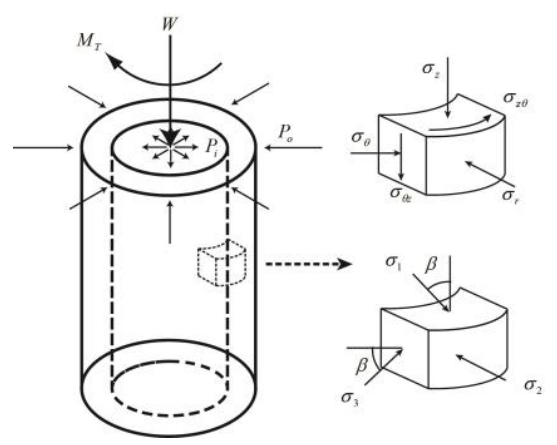

Fig.1. Stress state in hollow cylinder torsional shear test
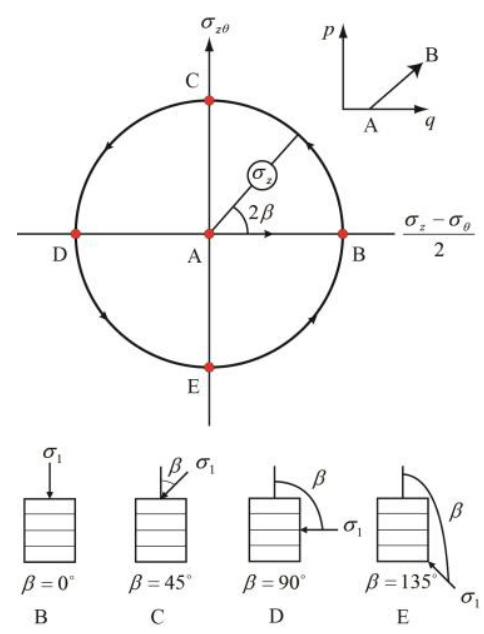

Fig.2. Principal stress rotation in deviatoric stress space

\subsection{Anisotropic Peak Stress Ratio $M_{\alpha}$}

Following Yao and Kong (2012), the following anisotropic peak stress ratio for anisotropic sand is defined:

$$
M_{\alpha}=M_{h}+\left(M_{v}-M_{h}\right) \frac{\alpha-\alpha_{h}}{\alpha_{v}-\alpha_{h}}
$$

The definition has been based on the consideration that the peak strength $(q / p)$ of an anisotropic soil is dependent not only on the stress condition but also on the minimum angle ( $\alpha$ in Fig.3) between the SMPs and the depositional planeat failure. For a given anisotropic soil, the internal friction angles $\varphi_{v}$ and $\varphi_{h}$ can be determined using conventional triaxial compression tests for vertically and horizontally layered specimens. For details please refer to Yao and Kong (2012).

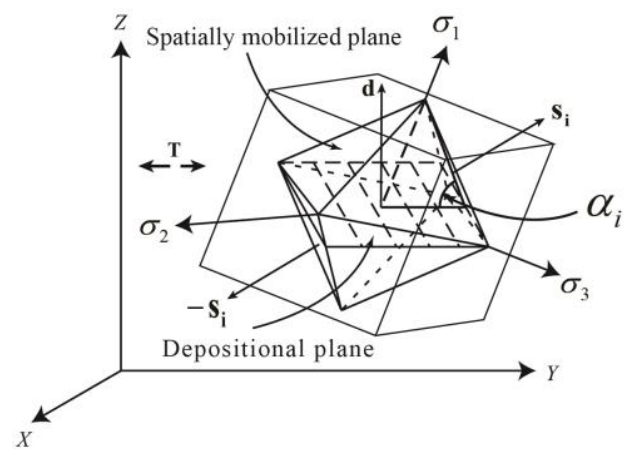

Fig.3. The relationship between SMPs and depositional plane in an anisotropic soil element (Yao and Kong, 2012)

\subsection{Transformed Anisotropic Stress Tensor}

A transformed stress tensor has been employed by Matsuoka et al. (1999) to transform the SMP (spacial mobilized plane) criterion into a circular shape in the transformed $\pi$-plane. Yao and Kong (2012) have further revised the transformed stress tensor by incorporating the anisotropic peak stress ratio $M_{\alpha}$. In the transformed stress space, the four new stress components, $\tilde{\sigma}_{z}, \tilde{\sigma}_{r}, \tilde{\sigma}_{\theta}$, and $\tilde{\sigma}_{z \theta}$ can be expressed as

$$
\begin{gathered}
\tilde{\sigma}_{z}=p+\frac{M}{M_{\alpha}} \frac{q^{*}}{q}\left(\sigma_{z}-p\right) \\
\tilde{\sigma}_{r}=p+\frac{M}{M_{\alpha}} \frac{q^{*}}{q}\left(\sigma_{r}-p\right) \\
\tilde{\sigma}_{\theta}=p+\frac{M}{M_{\alpha}} \frac{q^{*}}{q}\left(\sigma_{\theta}-p\right) \\
\tilde{\sigma}_{z \theta}=\frac{M}{M_{\alpha}} \frac{q^{*}}{q} \sigma_{z \theta}
\end{gathered}
$$

where $M$ is the peak stress ratio for the equivalent isotropic material in the transformed stress space (Yao and Kong 2012). The mean principal stress $p$, the deviatoric stresses $q$, and $q^{*}$ are respectively defined as follows

$$
\begin{gathered}
p=\frac{\sigma_{z}+\sigma_{r}+\sigma_{\theta}}{3} \\
q=\sqrt{I_{1}^{2}-3 I_{2}} \\
q^{*}=\frac{2 I_{1}}{3 \sqrt{\left(I_{1} I_{2}-I_{3}\right) /\left(I_{1} I_{2}-9 I_{3}\right)}-1}
\end{gathered}
$$

In which $I_{1}, I_{2}$ and $I_{3}$ are respectively the first, second and third stress invariants of the conventional stress tensor

$$
\begin{aligned}
& I_{1}=\sigma_{1}+\sigma_{2}+\sigma_{3} \\
& I_{2}=\sigma_{1} \sigma_{2}+\sigma_{2} \sigma_{3}+\sigma_{3} \sigma_{1} \\
& I_{3}=\sigma_{1} \sigma_{2} \sigma_{3}
\end{aligned}
$$


Here $\sigma_{1}, \sigma_{2}$ and $\sigma_{3}$ are the principal stress components which are kept constant during a pure principal stress rotation test.

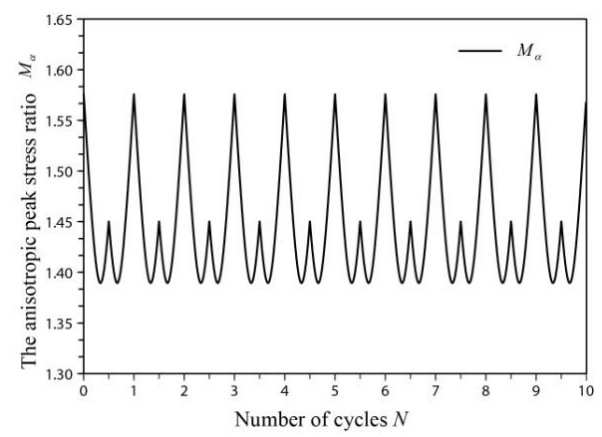

Fig.4. Changing of $M_{\alpha}$ with the rotation cycles

Two factors, the principal stress direction and its relative orientation with respect to the bedding plane of the anisotropic soil, need to be considered in determining the anisotropic variable $M_{\alpha}$. As shown in Fig. 4, the anisotropic peak stress ratio $M_{\alpha}$ changes cyclically in pure principal stress rotation tests. This is because the directional cosines of the SMPs also change cyclically with the cyclic rotation of the principal stress directions.

Under cyclic rotational shearing condition, the mean principal stress $\tilde{p}$ and the deviatoric stress $\tilde{q}$ in the new transformed stress space can be defined as

where

$$
\begin{gathered}
\tilde{p}=\frac{\tilde{\sigma}_{z}+\tilde{\sigma}_{r}+\tilde{\sigma}_{\theta}}{3} \\
\tilde{q}=\sqrt{\tilde{I}_{1}^{2}-3 \tilde{I}_{2}}
\end{gathered}
$$
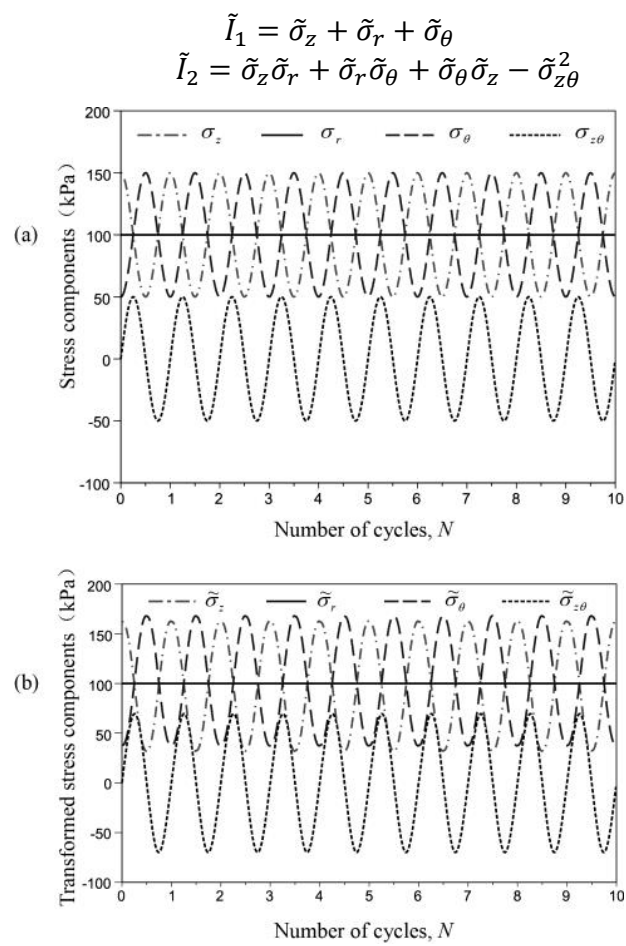

Fig.5. Stress paths of pure rotational shear: (a) in the original stress space; and (b) in the transformed stress space

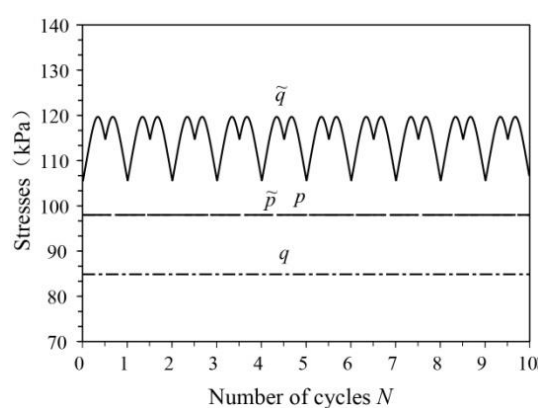

Fig.6. Stresses $p, q, \tilde{p}$ and $\tilde{q}$ versus the rotation cycles

$M_{\alpha}$ varies cyclically with the stress rotation, so are the transformed stresses. Fig.5 (a) shows the initial stress path under pure principal stress rotation, and Fig.5 (b) shows the stress path in the new transformed stress space of the same sample. It is evident from the two figures that the amplitudes of $\widetilde{\sigma}_{z}, \tilde{\sigma}_{\theta}$ and $\tilde{\sigma}_{z \theta}$ become greater than those of $\sigma_{z}, \sigma_{\theta}$ and $\sigma_{z \theta}$, while $\tilde{\sigma}_{r}$ and $\sigma_{r}$ are kept identical and constant. Fig.6 further shows the variation of $\mathrm{p}, \mathrm{q}, \tilde{p}$ and $\tilde{q}$ with the cyclic number. It is readily seen that the mean principal stresses $p, \tilde{p}$, and the deviatoric stress $q$ remain constant during the cyclic loading course while the deviatoric stress $\tilde{q}$ varies cyclically with the rotation of the principal stress axes.

\section{MODIFIED UH MODEL FOR CYCLIC LOADING}

Based on the transformed anisotropic stresses defined in last section, we are ready to modify the UH model in Yao and Kong (2012) for cyclic loading case. Following the theory of plasticity, the total strain increment is decomposed into elastic and plastic parts.

\subsection{Elastic relation}

The incremental elastic relation in the Cam-clay model is modified to include the transformed stress tensor as follows to describe the elastic behavior of the soil

$$
d \varepsilon_{i j}^{e}=\frac{1+v}{E} d \tilde{\sigma}_{i j}-\frac{v}{E} d \tilde{\sigma}_{m m} \delta_{i j}
$$

where $v$ is the Poisson's ratio, $E$ is the elastic module, and $\delta_{i j}$ is the Kronecker delta. The elastic module $E$ is dependent upon the mean principal stress $p$

$$
E=\frac{3(1-2 v)\left(1+e_{0}\right)}{\kappa} p
$$

where $e_{0}$ is the initial void ratio, and $\kappa$ is the slope of isotropic unloading curve in the $e-\ln p$ plane.

\subsection{Plastic relation}

As shown in Fig.7, two yield surfaces, the current yield surface and the reference yield surface, are employed in this paper which present a similar shape to the Cam-clay yield surface. They are plotted in the transformed stress space. In the figure $\mathrm{A}(\tilde{p}, \tilde{q})$ denotes a stress point on the current yield surface, while 
$B(\tilde{\bar{p}}, \tilde{\bar{q}})$ represent the corresponding mapping point of $A$ on the reference yield surface. The mapping follows a simple rule of constant stress ratio, such that $\tilde{\eta}=$ $\tilde{q} / \tilde{p}=\tilde{\bar{q}} / \tilde{\bar{p}}$.

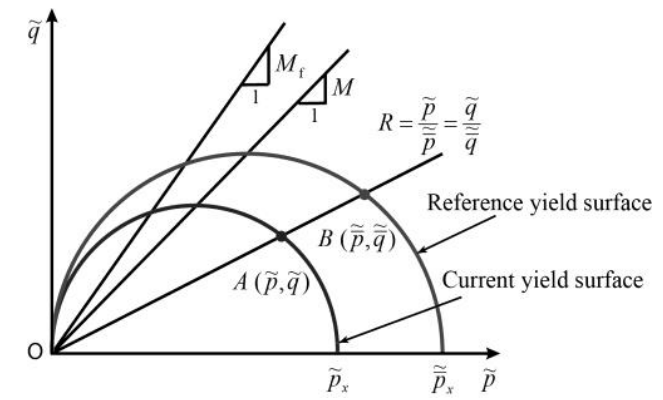

Fig.7. Reference yield surface and current yield surface in $\tilde{p}-\tilde{q}$ space

The plastic volumetric strain $\varepsilon_{v}^{p}$ is chosen as the hardening parameter. The reference yield surface adopts an expression of the following form

$$
\tilde{\tilde{f}}=\ln \frac{\tilde{\bar{p}}}{\tilde{\bar{p}}_{0}}+\ln \left[1+\frac{\overline{\tilde{q}}^{2}}{M^{2} \tilde{\tilde{p}}^{2}}\right]-\frac{\varepsilon_{v}^{p}}{c_{p}}=0
$$

where $\tilde{\bar{p}}_{0}$ is the preconsolidation pressure; $c_{p}=(\lambda-\kappa) /\left(1+e_{0}\right)$.

An associated flow rule is adopted here. The following identical form of yield function $\tilde{f}$ and plastic potential function $\tilde{g}$ is employed in terms of the anisotropic transformed stresses

$$
\tilde{f}=\tilde{g}=\ln \frac{\tilde{p}}{\tilde{p}_{x}}+\ln \left[1+\frac{\tilde{q}^{2}}{M^{2} \tilde{p}^{2}}\right]=0
$$

Where $\tilde{p}_{x}$ is the intersection of the current yield surface with the $\tilde{p}$-axis. Important to the $\mathrm{UH}$ model in Yao and Kong (2012) extended is the introduction of an anisotropic unified hardening parameter $H$, which is defined in terms of the potential failure stress ratio $M_{f}$ and the equivalent peak stress ratio $M$ :

$$
H=\int d H=\int \frac{M_{f}^{4}-\tilde{\eta}^{4}}{M^{4}-\tilde{\eta}^{4}} d \varepsilon_{v}^{p}
$$

where $\tilde{\eta}=\tilde{q} / \tilde{p}$. The potential failure stress ratio for sands is defined by

$$
M_{f}=6\left(\sqrt{\frac{k}{\widetilde{R}^{m}}\left(1+\frac{k}{\widetilde{R}^{m}}\right)}-\frac{k}{\widetilde{R}^{m}}\right)
$$

where $k=M^{2} /(12(3-M)), m$ is a parameter for sand which reflects the effect of $\tilde{R}$ on soil strength, and $\tilde{R}$ is the overconsolidation (OC) parameter (Yao et al. 2009),

$$
\tilde{R}=\frac{\tilde{p}}{\tilde{\bar{p}}_{x 0}}\left(1+\frac{\tilde{\eta}^{2}}{M^{2}}\right) \exp \left(-\frac{\varepsilon_{v}^{p}}{c_{p}}\right)
$$

By using the same $H$, Eq. (23) can be recast as

$$
\tilde{f}=\ln \frac{\tilde{p}}{\tilde{p}_{0}}+\ln \left[1+\frac{\tilde{q}^{2}}{M^{2} \tilde{p}^{2}}\right]-\frac{H}{c_{p}}=0
$$

The plastic strains are controlled by the current stresses at yield and can be given by

$$
d \varepsilon_{i j}^{p}=\Lambda \frac{\partial \tilde{f}}{\partial \tilde{\sigma}_{i j}}
$$

where $\Lambda$ is the plastic factor obtained by imposing the condition of consistency

$$
\Lambda=\frac{M^{4}-\tilde{\eta}^{4}}{M_{f}{ }^{4}-\tilde{\eta}^{4}} c_{p}\left(d \widetilde{p}+\frac{2 \tilde{p} \tilde{q}}{M^{2} \tilde{p}^{2}-\tilde{q}^{2}} d \tilde{q}\right)
$$

It is assumed that the stresses are coaxial with plastic strain increments, and anisotropy affects only the dilatancy and plastic modulus but not the plastic strain increment direction in the transformed stress space. As a result, the vertical, radial, and circumferential and shear strain increments can be determined from

$$
\begin{aligned}
& d \varepsilon_{z}^{p}=\Lambda \frac{\partial \tilde{f}}{\partial \tilde{\sigma}_{z}}, \quad d \varepsilon_{r}^{p}=\Lambda \frac{\partial \tilde{f}}{\partial \tilde{\sigma}_{r}}, \\
& d \varepsilon_{\theta}^{p}=\Lambda \frac{\partial \tilde{f}}{\partial \tilde{\sigma}_{\theta}}, \quad d \varepsilon_{z \theta}^{p}=\Lambda \frac{\partial \tilde{f}}{\partial \tilde{\sigma}_{z \theta}}
\end{aligned}
$$

where

$$
\begin{gathered}
\frac{\partial \tilde{f}}{\partial \tilde{\sigma}_{z}}=\frac{1}{M^{2} \tilde{p}^{2}+\tilde{q}^{2}}\left[\frac{M^{2} \tilde{p}^{2}-\tilde{q}^{2}}{3 \tilde{p}}+3\left(\tilde{\sigma}_{z}-\tilde{p}\right)\right] \\
\frac{\partial \tilde{f}}{\partial \tilde{\sigma}_{r}}=\frac{1}{M^{2} \tilde{p}^{2}+\tilde{q}^{2}}\left[\frac{M^{2} \tilde{p}^{2}-\tilde{q}^{2}}{3 \tilde{p}}+3\left(\tilde{\sigma}_{r}-\tilde{p}\right)\right] \\
\frac{\partial \tilde{f}}{\partial \tilde{\sigma}_{\theta}}=\frac{1}{M^{2} \tilde{p}^{2}+\tilde{q}^{2}}\left[\frac{M^{2} \tilde{p}^{2}-\tilde{q}^{2}}{3 \tilde{p}}+3\left(\tilde{\sigma}_{\theta}-\tilde{p}\right)\right] \\
\frac{\partial \tilde{f}}{\partial \tilde{\sigma}_{z \theta}}=\frac{3 \tilde{\sigma}_{z \theta}}{M^{2} \tilde{p}^{2}+\tilde{q}^{2}}
\end{gathered}
$$

The plastic volumetric strain increment can be obtained by

$$
d \varepsilon_{v}^{p}=d \varepsilon_{z}^{p}+d \varepsilon_{r}^{p}+d \varepsilon_{\theta}^{p}
$$

From Eq. (23) we have

$$
\begin{gathered}
\tilde{p}_{x}=\tilde{p}\left(1+\frac{\tilde{q}}{M^{2} \tilde{p}^{2}}\right) \\
d \widetilde{p}_{x}=\frac{2 \tilde{q}}{M^{2} \tilde{p}} d \widetilde{q}
\end{gathered}
$$

The loading conditions are specified as follows

$$
\left\{\begin{array}{c}
\text { Loadingoccurswhen } d \tilde{p}_{x}>0 ; \text { and } d \widetilde{p}_{x} \leq 0 \text { with } \tilde{\eta}>M_{f} \\
\text { Neutralloadingoccurswhen } d \widetilde{p}_{x}=0 \\
\text { Unloadingoccurswhen } d \widetilde{p}_{x}<0
\end{array}\right.
$$

\section{MODEL VERIFICATION}

Drained tests for Toyoura standard sand with inherent fabric anisotropy were performed on an 
automatic hollow cylinder apparatus by Miura et al. (1986) and Tong et al. (2010). The samples were subjected to cyclic rotation of principal stress axes while the magnitudes of effective principal stresses were maintained constant. The contractive volumetric strain was found to accumulate progressively by a decreasing accumulation rate with the increasing number of rotation cycles $N$.

Table 1. Summary of the constitutive parameters for Toyoura standard sand

\begin{tabular}{clc}
\hline Parameter & Physical meaning & value \\
\hline$\lambda$ & slope of normal compression line & 0.012 \\
\hline$\kappa$ & slope of unloading line & 0.0035 \\
\hline$V$ & Poisson's ratio & 0.15 \\
\hline$e_{0}$ & initial void ratio & 0.74 \\
\hline$m$ & $\begin{array}{l}\text { parameter reflecting the effect of } \tilde{R} \\
\text { on soil strength }\end{array}$ & 1.5 \\
\hline$\tilde{R}_{i}$ & $\begin{array}{l}\text { initial value of the overconsolidation } \\
\text { parameter } \tilde{R}\end{array}$ & 1.0 \\
\hline$\varphi_{v}$ & $\begin{array}{l}\text { friction angle at critical state of } \\
\text { vertical specimen }\end{array}$ & $31.0^{\circ}$ \\
\hline$\varphi_{h}$ & $\begin{array}{l}\text { friction angle at critical state of } \\
\text { horizontal specimen }\end{array}$ & $28.6^{\circ}$ \\
\hline$\varphi_{f v}$ & $\begin{array}{l}\text { friction angle at failure of vertical } \\
\text { specimen }\end{array}$ & $39.5^{\circ}$ \\
\hline$\varphi_{f h}$ & $\begin{array}{l}\text { friction angle at failure of horizontal } \\
\text { specimen }\end{array}$ & $36.5^{\circ}$ \\
\hline
\end{tabular}

The constitutive model needs ten parameters to be calibrated. Among them, $\lambda$ and $\kappa$ are the slopes of void ratio versus natural logarithm of effective volumetric stress during virgin loading and reloading of samples under isotropic consolidation conditions, respectively, as the same in Cam-clay model. $\varphi_{f v}, \varphi_{f h}$, $\varphi_{v}$ and $\varphi_{h}$ can be determined from conventional compression tests for vertical samples and horizontal samples, respectively. Table 1 summarizes the values that have been calibrated for Toyoura sand.

Presented in Fig. 8 are the test results by Miura et al. (1986) and the predictions by the proposed model for the first rotation cycle. It can be seen that the predicted strains agree well with the trend of the experimental results. The impact of fabric anisotropy on the response of sand can be clearly captured by the present model.

The test data reported by Tong et al. (2010) have been further used to validate our model. Figure 9 and 10 compare the measured and the predicted volumetric strain of Toyoura standard sand during the loading course of drained pure rotational shearing for 17 cycles. It can be seen from Fig. 9 and Fig. 10 that the model correctly predicts the dominant contraction behavior observed in the tests on the volumetric deformation caused by pure principal stress axes rotation. While the volumetric strain increases with the rotation cycle, its accumulation rate decreases steadily with the increase of $N$. The trend agrees well with experimental observations as shown in the simulations. (a)

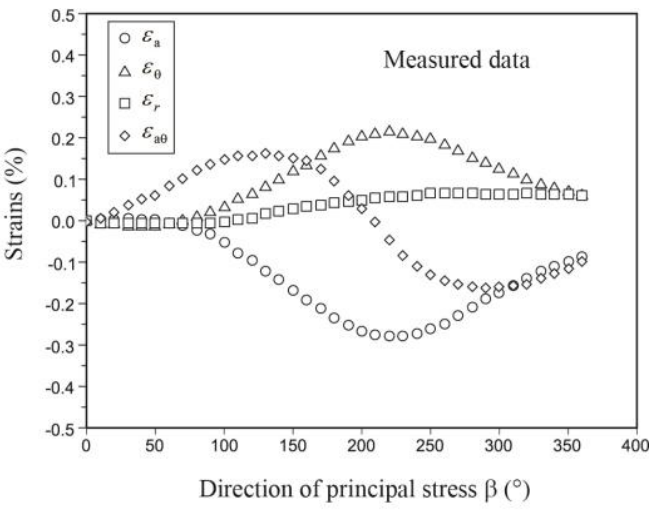

(b)

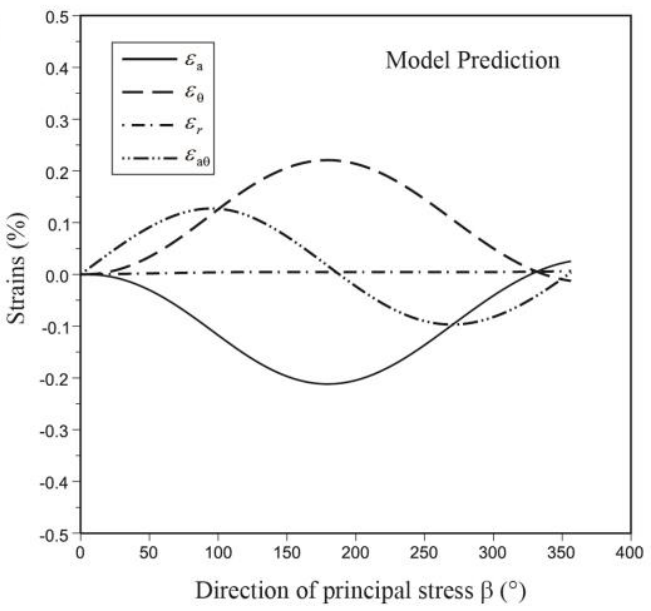

Fig.8. Comparison of measured and predicted relationships between strains and direction of principal stress (Data after Miura et al. 1986)

(a)

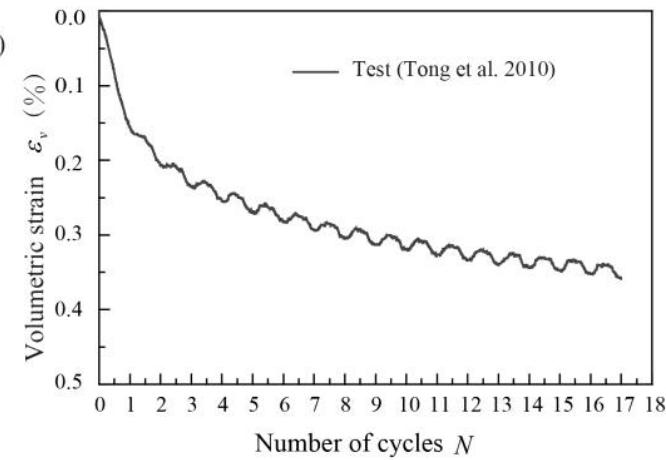

(b)

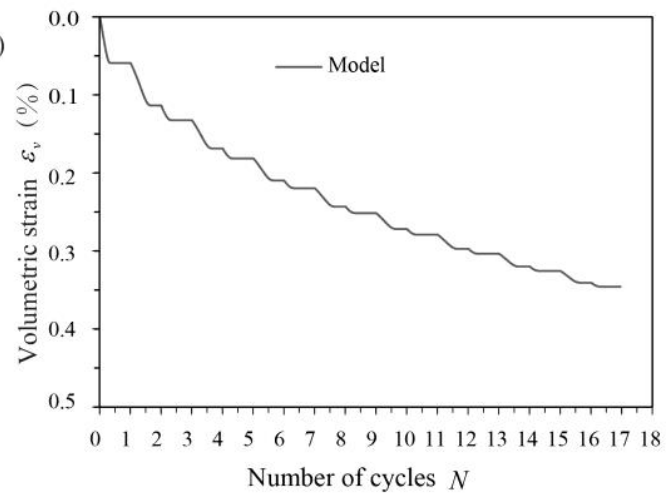

Fig.9. Measured and predicted volumetric strain for drained pure rotational shear tests on Toyoura standard sand (Data after Tong et al. 2010). 
(a)

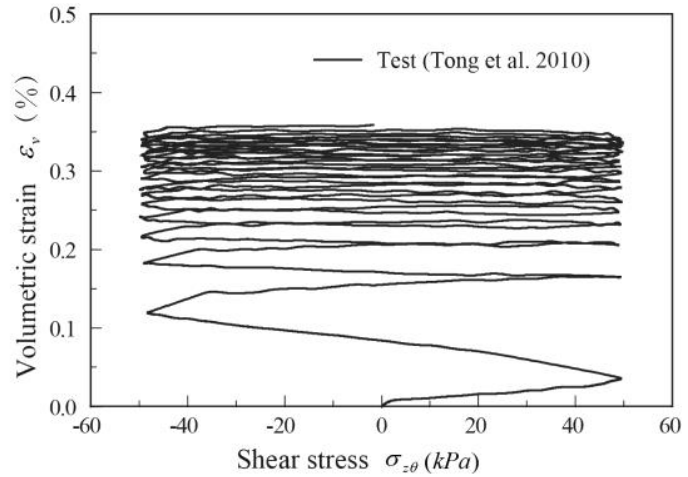

(b)

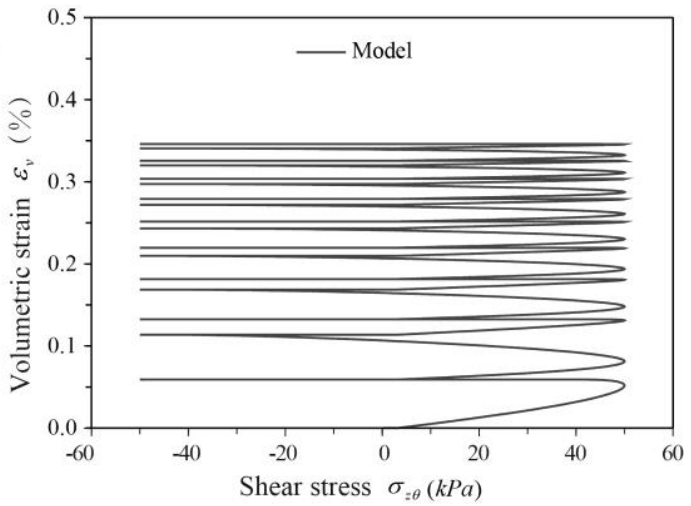

Fig.10 Measured and predicted volumetric strain versus shear stress for drained pure rotational shear tests on Toyoura standard sand (Data after Tong et al. 2010).

\section{CONCLUSION}

A constitutive model has been presented in this study to simulate the behavior of anisotropic sand subjected to cyclic loading with principal stress rotation. The model is formulated on basis of a previous one proposed by Yao and Kong (2012), by incorporating a newly defined transformed anisotropic stress tensor, and a new parameter $m$ which reflects the effect of the overconsolidation parameter $\tilde{R}$ on sand strength. In the constitutive model the plastic strain increments are directly related the transformed stress increments. Ten model parameters are required in the constitutive model to fully characterize the sand behavior under principal stress rotation. To examine the influence of principal stress rotation on the soil behavior, test results of anisotropic Toyoura standard sand are compared with predictions of the proposed constitutive model. The model is shown to be capable of characterizing the typical behavior observed in experimental tests on anisotropic sand subjected to rotational shearing. The effect of inherent anisotropy on the cyclic behavior of sand is well captured.

\section{ACKNOWLEDGEMENTS}

This work was supported by the National Natural Science Foundation of China (Grant No.11402109) and the Natural Science Foundation of Jiangsu Province (Grant No.BK20130909).

\section{REFERENCES}

1) Arthur, J., Chua, K., and Dunstan, T. (1979). Dense sand weakened by continuous principal stress direction rotation. Geotechnique, 29(1), 91-98.

2) Grabe, P. J. and Clayton, C. (2009). Effects of principal stress 207 rotation on permanent deformation in rail track foundations. Journal of Geotechnical and Geoenvironmental Engineering, 135(4), 555-565.

3) Hicher, P. Y. and Lade, P. V. (1987). Rotation of principal directions in k0-consolidated clay. Journal of Geotechnical Engineering, 113(7), 774-789.

4) Hight, D.W., Gens, A., and Symes, M. J. (1983). The development of a new hollow cylinder apparatus for investigating the effects of principal stress rotation in soils. Geotechnique, 33(4), 355-383.

5) Ishihara, K. and Towhata, I. (1983). Sand response to cyclic rotation of principal stress directions as induced by wave loads. Soils and Foundations, 23(4), 11-26.

6) Ishihara, K. and Yamazaki, A. (1984). Analysis of wave-induced liquefaction in seabed deposits of sand. Soils and Foundations, 24(3), 85-100.

7) Matsuoka, H. (1974). Stress-strain relationship of sands based on the spatial mobilized plane. Soils and Foundations, 14(2), 47-61.

8) Matsuoka, H. (1976). On the significance of the spatial mobilized plane. Soils and Foundations, 16(1), 91-100.

9) Matsuoka, H., Yao, Y., and Sun, D. (1999). The Cam-clay models revised by the SMP criterion. Soils and Foundations, 39(1), 81-95.

10) Miura, K., Miura, S., and Toki, S. (1986). Deformation behavior of anisotropic dense sand under principal stress axes rotation. Soils and foundations, 26(1), 36-52.

11) Nakata, Y., Hyodo, M., Murata, H., and Yasufuku, N. (1998). Flow deformation of sands subjected to principal stress rotation. Soils and Foundations, 38(2), 115-128.

12) Sivathayalan, S. and Vaid, Y. P. (2002). Influence of generalized initial state and principal stress rotation on the undrained response of sands. Canadian Geotechnical Journal, 39(1), 63-76.

13) Symes, M., Gens, A., and Hight, D. W. (1984). Undrained anisotropy and principal stress rotation in saturated sand.. Geotechnique, 34(1), 11-27.

14) Symes, M. J., Gens, a., and Hight, D. W. (1988). Drained principal 234 stress rotation in saturated sand. Geotechnique, 38(1), 59-81.

15) Tong, Z.-X., Zhang, J.-M., Yu, Y.-L., and Zhang, G. (2010). Drained deformation behavior of anisotropic sands during cyclic rotation of principal stress axes. Journal of Geotechnical and Geoenvironmental Engineering, 136(11), $1509-1518$

16) Vaid, Y. P., Sayao, A., Hou, E., and Negussey, D. (1990). Generalized stress-path-dependent soil behaviour with a new hollow cylinder torsional apparatus. Canadian Geotechnical Journal, 27(5), 601-616.

17) Yang, Z. X., Li, X. S., and Yang, J. (2007). Undrained anisotropy and rotational shear in granular soil. Geotechnique, 57(4), 371-384.

18) Yao, Y. P., Hou, W., and Zhou, A. N. (2009). UH model: three-dimensional unified hardening model for overconsolidated clays. Geotechnique, 59(5), 451-469.

19) Yao, Y. P. and Kong, Y. X. (2012). Extended uh model: Three-dimensional unified hardening model for anisotropic clays. Journal of Engineering Mechanics, 138(7), 853-866. 